\title{
Correction to: Explicit Third-Order Unconditionally Structure-Preserving Schemes for Conservative Allen-Cahn Equations
}

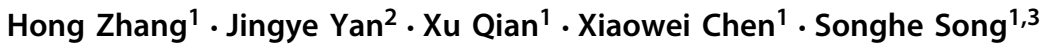 \\ Published online: 23 February 2022 \\ ๑) Springer Science+Business Media, LLC, part of Springer Nature 2022
}

\section{Correction to: Journal of Scientific Computing (2022) 90:8 https://doi.org/10.1007/s10915-021-01691-w}

The original version of the article unfortunately contained a mistake.

The enunciation label "Theorem 3.6" on Equation [35] were removed i, and the remaining references were renumbered mistakenly. It has been corrected in this correction.

1. The Theorem 3.6 should be added in the sentence starts with "Assume the underlying...." above the equation (35). It should be

Theorem 3.6Assume the underlying RK Butcher tableau satisfy the third condition in Assumption 3.1, then the isIFRK scheme (28)conserve the mass of (21),i.e.,

2. The Theorem 3.6 should be changed to Theorem 3.7 under the heading " 3.4 Numerical Analysis".

Theorem 3.7We assume that $\boldsymbol{u}(t) \in C^{p}[0, T]$ is the exact solution to the space-discrete system (18) and $\boldsymbol{u}^{n}$ is the solution to isIFRK schemes, respectively. We further assume that the

The original article can be found online at https://doi.org/10.1007/s10915-021-01691-w.

$\varangle \quad$ Xu Qian

qianxu@nudt.edu.cn

Hong Zhang

zhanghnudt@163.com

Jingye Yan

yanjingye0205@163.com

Xiaowei Chen

cwx@nudt.edu.cn

Songhe Song

shsong@nudt.edu.cn

1 Department of Mathematics, College of Liberal Arts and Science, National University of Defense Technology, Changsha 410073, Hunan, China

2 Division of Mathematical Sciences, Nanyang Technological University, Singapore 637371, Singapore

3 State Key Laboratory of High Performance Computing, National University of Defense Technology, Changsha 410073, China 
initial value is smooth and satisfies $\left\|\boldsymbol{u}_{0}\right\|_{l} \infty \leq \beta$. Then we have the following error estimates for the isIFRK schemes (31-33) with pth-order underlying Butcher tableau as

Publisher's Note Springer Nature remains neutral with regard to jurisdictional claims in published maps and institutional affiliations. 